\title{
Breve roteiro da investigação empírica na Pedagogia do Desporto: a investigação sobre o ensino da educação física
}

\author{
Amândio Graça \\ Faculdade de Ciências do Desporto e de Educação Física, \\ Universidade do Porto \\ https://doi.org/10.5628/rpcd.01.01.104
}

\begin{abstract}
A QUESTÃO DA EFICÁCIA DO ENSINO
A investigação sobre o ensino, qualquer que seja o seu modo de se posicionar ou a sua orientação conceptual ou metodológica, está intrinsecamente imbuída de interesse pela melhoria das práticas de ensino e aprendizagem (82), mesmo se esse interesse não se apresenta explicitamente declarado ou perspectivado para o imediato. A eficácia do ensino será um daqueles temas que por certo há-de acompanhar os caminhos da História da Educação. A ideia da excelência na acção educativa está presente nas sucessivas fases por que tem passado a investigação sobre a eficácia do ensino $(3,20,33,46,53,84)$. As mudanças de fase são assinaladas pela mudança do local de focagem da questão central. Mas acontece que as questões são frequentemente recorrentes. Fases seguintes recuperam ou preservam elementos de fases anteriores. As velhas questões passam por vezes para um segundo ou terceiro plano, ou podem reaparecer reformuladas e sobretudo iluminadas por novos modos de olhar, quer dizer, novos conceitos e novas metodologias. Este facto pode sugerir a presença de um efeito de 'moda' na ascensão e declínio dos paradigmas de investigação.
\end{abstract}

\section{AS CARACTERÍSTICAS DO BOM PROFESSOR}

A questão da $1^{\text {a }}$ fase de investigação do ensino geral, que prevaleceu durante a primeira metade do século XX (23), centrou-se sobre as características do bom professor. Medidas do QI e testes de personalidade; listas de características do bom professor, segundo a opinião dos alunos; e avaliações impressionistas de directores escolares ou professores foram usados para categorizar professores e permitir a comparação dos resultados dos alunos em testes estandardizados ou em exames. Muitos destes estudos padeciam de evidentes fragilidades conceptuais e metodológicas e, genericamente, os seus resultados foram pouco animadores (30):

a) Os testes de personalidade não dizem grande coisa a respeito de como o professor se assume e se comporta na sua actividade pedagógica e na sua relação com os alunos ${ }^{(61,65)}$;

b) as apreciações globais e subjectivas de inspectores, directores da escola ou professores, muitas das vezes sem sequer terem observado o professor a ensinar, não oferecem qualquer garantia de validade e fidelidade ${ }^{(84)}$;

c) os professores que possuíam mais características da lista do bom professor não obtiveram em geral e de forma consistente maiores ganhos de aprendizagem com os seus alunos ${ }^{(53)}$;

d) as características do professor, ainda que muito plausivelmente importantes, não actuarão isoladamente.

Este programa de estudo não teve grande eco na área da educação física (50).

\section{A ELEIÇÃO DO MELHOR MÉTODO}

A $2^{\text {a }}$ fase centrou-se sobre a descoberta do método ideal, através da comparação de resultados produzidos pela aplicação de diferentes métodos de ensino.

À pouca credibilidade académica e científica generalizadamente atribuída à sistematização do conhecimento sobre os métodos de ensino, procurou-se responder com a exibição dos pergaminhos da prova científica e do método 
experimental clássico. Métodos de ensino derivados de teorias psicológicas ou extrapolados de descobertas de estudos laboratoriais da aprendizagem motora foram ensaiados e testados em situações de ensino artificiais ou muito condicionadas. Os resultados obtidos por este tipo de pesquisa mostraram-se, no entanto, inconclusivos, contraditórios e por vezes, ingénua ou suspeitosamente tendenciosos. Do ponto de vista conceptual havia alguma confusão entre ensino e aprendizagem motora. Isso mesmo está patente no capítulo de revisão que Nixon e Locke (55) escreveram para o $2^{\circ}$ volume do Handbook of Research on Teaching. Por outro lado, do ponto vista metodológico, a ausência de controlo das variáveis de processo, dada a inexistência de indicadores de comprovação da implementação do método (51), o número reduzido de turmas incluídas nos estudos e a adopção de unidades de análise inadequadas confundiam os efeitos eventualmente produzidos pelos métodos com efeitos atribuíveis a diferenças idiossincráticas dos professores (7). Este esquema de investigação, foi muito popular na Educação Física, pois oferecia um esquema expedito para resolver os problemas pessoais da elaboração das provas académicas, mas não tinha força para se transformar num programa credível e produtivo. $\mathrm{O}$ interesse pelos métodos não desapareceu, antes pelo contrário, mas foi obrigado a procurar legitimidade no quadro das condições reais em que o ensino ocorre. Desta forma o interesse pelos métodos sobrevive à fase seguinte da investigação, revigorando-se nela, ou então afirmando-se em contraponto. Em vez de continuarmos a perguntar qual é o melhor método, Rink (63) aconselha-nos antes a questionarmo-nos "para que propósitos, em que circunstâncias e de que maneira devo eu utilizar esta metodologia de instrução?” (p. 9).

O comportamento do professor eficaz

A questão central da $3^{\text {a }}$ fase (com início na década de 60) foi a de saber o que faz o professor eficaz. Como é que os comportamentos de ensino, as variáveis de processo observadas na aula se associavam aos resultados da aprendizagem dos alunos, as variáveis de produto. O propósito era o de isolar e identificar uma lista de competências do professor eficaz. Dunkin e Biddle (22) forneceram um modelo conceptual robusto que facilitava a integração do delineamento dos projectos de pesquisa individuais num programa geral de investigação. O modelo permitia considerar na análise do ensino a multiplicidade de relações possíveis entre factores de diversa ordem: variáveis do contexto (do aluno, da escola e da comunidade), variáveis de presságio (personalidade, formação e experiência dos sujeitos do estudo, professores ou alunos), variáveis de processo (comportamentos do aluno, comportamentos de ensino) e variáveis de produto. $\mathrm{O}$ paradigma processo-produto estabeleceu o desenvolvimento da sua investigação num encadeamento de 3 momentos - descriptivecorrelational-experimental loop (66): O momento descritivo dá resposta ao estudo exploratório e de fecundidade das categorias de observação; o momento correlacional estabelece a associação entre as variáveis de processo e os ganhos de aprendizagem das turmas com bons ou maus níveis de rendimento; o momento experimental treina implementa e testa programas de aplicação das variáveis de processo previamente associadas aos maiores ganhos de aprendizagem.

Vários estudos replicaram correlações consistentes entre variáveis de processo e rendimento dos alunos. Os princípios e modelos estabelecidos com base na investigação tiveram viabilidade experimental $(7,67,54)$. Dado terem sido geradas nas condições reais de ensino, e não a partir de situações de laboratório, as descobertas foram percebidas como tendo grande utilidade para a melhoria das escolas, para a avaliação do ensino e para a definição dos programas da formação de professores. A sua influência estendeu-se a diversos níveis do aparelho do sistema educativo.

Da síntese dos grandes estudos processo-produto emergiu o modelo de Direct Instruction. A economia do modelo é evidente. Housner (42) sistematiza-o em 5 alíneas:

a) definir objectivos claros e garantir que os alunos os compreendem;

b) apresentar uma sequência de tarefas académicas bem organizadas;

c) fornecer aos alunos explicações claras e concisas da matéria, que incluam coisas como a utilização 
liberal de demonstrações e ilustrações;

d) colocar perguntas frequentes de modo a controlar a compreensão dos alunos e reensinar se necessário;

e) proporcionar aos alunos frequentes oportunidades de sucesso nas tarefas académicas e disponibilizar feedback.

Mas, apesar do sucesso do programa de investigação na satisfação dos objectivos a que se propusera, ele entra em perda da posição dominante nos anos 80 , no ensino geral e 10 anos mais tarde na educação física, assistindo-se a um deslocamento da agenda para outros programas e paradigmas de investigação. Aquilo que era inicialmente a sua grande vantagem, a utilização de instrumentos de observação objectivos e sistemáticos passou a ser criticado com base no argumento de que a agregação descontextualizada dos comportamentos e o recurso a variáveis de baixa inferência nos procedimentos de recolha de dados comprometerem a consideração da intencionalidade do ensino. Não respeitando as relações de fronteira do comportamento com as condições situacionais em que ocorre era inevitável a perda de acesso aos propósitos das acções (20). Outra das críticas mais fortes ao paradigma processo-produto é o da sua propensão para o ateoricismo, que se evidenciaria tanto na ausência de uma perspectiva teórica para dar significado e coerência ao direccionamento das questões sobre o ensino, como na insuficiente integração dos resultados da investigação num esforço de teorização. A investigação processo-produto parece dar-se por satisfeita por dar conta do que funciona aparentemente bem, sem cuidar de saber porquê (82). Tornou-se evidente a insustentabilidade da assunção de uma relação directa e mecânica entre comportamento de ensino e resultados de aprendizagem. Entre o processo de ensino e o produto da aprendizagem do aluno há um espaço de explicação ausente ou insuficientemente conceptualizado.

A forma como os resultados da investigação processo-produto se repercutiram nas políticas de avaliação, promoção na carreira e formação de professores mereceu a reprovação inclusive das suas principais figuras $(7,54)$. Em vez de serem entendidas como princípios gerais de actuação que norteariam as decisões dos professores nas particularidades de ensino, as conclusões da investigação foram, em diversos locais, implementadas como prescrições estritas de comportamentos. Duas mensagens reducionistas, (1) a de que a competência pedagógica pode ser equacionada de forma clara e simples e (2) a de que as descobertas da investigação processoproduto fornecem a matéria essencial para a formação de professores, servem de pretexto à redução da duração da formação inicial, contribuem para o enfraquecimento do professor enquanto autor do seu ensino e reforçam a sua dependência do controlo externo de gestores, avaliadores e especialistas do currículo $(20,42)$.

\section{O COMPORTAMENTO DO ALUNO E A GESTÃO DO TEMPO DE AULA}

O Beginning Teacher Evaluation Study (BTES) constitui a primeira tentativa de resposta a este problema do hiato entre comportamento de ensino e resultado de aprendizagem. Com efeito, o Academic Learning Time (ALT), centrando-se estrategicamente sobre a actividade do aluno, veio a demonstrar-se como uma variável muito forte e com valor preditivo para a consecução dos alunos $(5,17,84)$. Ele marcou profundamente a agenda da investigação do ensino da Educação Física, desde os finais da década de 1970 até aos nossos dias, $(89,57,10)$. O Sistema de Observação ALT-PE (Academic Learning Time in Physical Education) desenvolvido por Siedentop, Birdwell e Metzler (87) e modificado por Siedentop, Tousignant e Parker (88) e o sistema OBEL-ULG (Observation de l'Éleve), desenvolvido por Piéron (58), ambos centrados na actividade do aluno e na gestão do tempo da aula, alimentaram grande parte da investigação no ensino da educação física. No entanto, o tempo continua a não satisfazer as exigências de explicação, por demasiado grosseiro, incapaz de diferenciar as nuances de qualidade no conteúdo que quer medir (82). Needels e Gage (54) procuram colmatar o problema da insuficiente contemplação das variáveis de mediação através da sugestão de uma melhor fundamentação teórica dos processos de aprendizagem com base nas concepções cognitivas da aprendizagem do aluno. Housner (42) sublinha a razão de ser dessa necessidade com a evidência empírica que aponta para um muito maior 
poder preditivo das estratégias cognitivas do aluno sobre os seus resultados escolares. A assunção implícita de uma causalidade linear simples entre comportamento de ensino e resultado da aprendizagem dá lugar a uma perspectiva bem mais complexa envolvendo a interacção dos comportamentos de ensino com os processos de mediação do aluno.

Rink (63) propõe que esta interacção entre eficácia do ensino, dos métodos de instrução, e a aprendizagem seja analisada através da perspectiva de uma teoria de aprendizagem.

A quantidade de tempo de empenhamento motor, o número de repetições ou o sucesso na realização das tarefas não pode ser abstraído da consideração fundamental que é o nível de processamento ou a qualidade do confronto do aluno com a tarefa de aprendizagem. Para Rink (63), se, quanto quando, e como fornecer informação ao aluno deve ser determinado em função do objectivo de estabelecer um nível óptimo de processamento:

Saber como pôr os alunos a processar o que estão a fazer quanto baste para "gerar" respostas motoras apropriadas e saber quando intervir com ajuda mais específica e diferentes tarefas que solicitem respostas mais avançadas é talvez a arte do ensino (p5).

\section{PERSPECTIVA ECOLÓGICA DA \\ AULA DE EDUCAÇÃO FÍSICA}

Os estudos ecológicos da sala de aula têm como primeira preocupação compreender os modos como o pensamento e acção são organizados pelas exigências do envolvimento $(18,19,20,21)$. Olha-se para a sala de aula como uma unidade ecocomportamental, composta por segmentos que circunscrevem e regulam os processos de pensamento e acção, tanto de professores como de alunos. (19, 20). Na Educação Física a perspectiva ecológica deu corpo a um programa de investigação na Universidade de Ohio, sob a liderança de Daryl Siedentop $(40,86)$. Este programa toma por referência a interacção e interdependência de três sistemas interrelacionados: o sistema de gestão, o sistema de instrução e o sistema de socialização dos alunos (91). Um conceito nuclear nestes estudos é o de accountability system, o sistema de exigências e responsabildades que opera na aula. Os primeiros estudos, de natureza etnográfica puseram em evidência algumas diferenças entre a ecologia do ensino geral e da educação física (93), salientando, nomeadamente a importância do sistema de socialização dos alunos, as manobras de modificação da actividade e de evitar a participação sem ser notado (competent bystander).

Os estudos da gestão da sala de aula lidam com a problemática da criação e manutenção da ordem na aula, e a garantia da cooperação dos alunos, aspectos essenciais para a viabilização do sistema de trabalho da turma, encarada na sua dimensão social (19). Os estudos em educação física revelam que os sistemas de gestão são melhor ensinados e supervisionados que os sistemas de instrução (86). As tarefas rotinadas ou facilmente rotináveis decorrem normalmente sem problemas, os alunos sabem funcionar nelas e sabem o que se espera que eles façam, que contas é que têm de prestar por elas. $\mathrm{O}$ lema do "happy, busy and good", destacado por Placek (59), ilustra a acomodação dos professores de Educação Física a uma actividade rotineira, ou ao fecho do negócio da tarefa pelo preço que os alunos bem quiserem dar. Ennis (26) reporta o abandono do currículo da instrução e a conformação com o currículo da manutenção da ordem e da motivação extrínseca. Em muitos casos a aula de educação física é dominada pelo sistema social dos alunos, transformando-se numa espécie de recreio supervisionado (86).

A linha de investigação mais centrada sobre a transacção académica tem como ponto de referência capital o conceito de tarefa. A tarefa refere-se à maneira como está definido o trabalho dos alunos e, por conseguinte, ao ajustamento do nível que se preconiza para o confronto dos alunos com as exigências académicas. Os conceitos de Rink (62) relacionados com a estrutura e sequência das tarefas de instrução (tarefas de informação, de extensão; de refinamento e de aplicação) permitiram estudar o funcionamento do sistema de tarefas na aula $(34,39,47)$. A interpretação das tarefas não é, porém, um processo linear. Com muita frequência, os alunos torneiam as tarefas ou inventam estratégias pessoais para despachar o trabalho, para as tornar mais divertidas (38), de tal forma que descaracterizam o objectivo preconizado para a tarefa. Em Educação 
Física é muito comum modificarem o grau de dificuldade da tarefa para reduzirem ou aumentarem o nível de desafio ou risco $(92,93)$. As operações preconizadas para a concretização das tarefas nem sempre são claramente explicitadas e algumas delas são mesmo intrinsecamente ambíguas. Decorre daqui um processo negocial mais ou menos subterrâneo que concretiza o leque de exigências das tarefas e que muitas das vezes redunda numa alteração completa da natureza da tarefa inicialmente preconizada pelo professor. Não raras vezes, tarefas apresentadas para trabalhar aspectos cognitivamente complexos vêem-se reduzidas a meros formalismos processuais.

\section{O PENSAMENTO DO PROFESSOR}

A afirmação veemente da natureza irredutivelmente complexa do processo de ensino-aprendizagem e o reconhecimento da insuficiência no entendimento do professor como um técnico que aplica com eficiência o know how do ensino aprofundam a perspectiva do professor como um profissional autónomo, tal como o médico, o advogado ou o arquitecto $(83,13,14)$, e legitimam a reivindicação de dar voz ao professor, o direito de ver considerada a sua perspectiva, o seu modo de ver as coisas nos seus próprios termos (20, 24). O paradigma do pensamento do professor coloca em primeiro plano a ligação entre os processos de pensamento e acção: A ideia de base é a de que aquilo que o professor faz é influenciado pelo que o professor pensa (15). Não sendo uma ideia revolucionária e fazendo parte até do esquema conceptual do processo de ensino e aprendizagem de Gage (82), o questionamento empírico do pensamento do professor só se torna viável com o crescimento da influência das perspectivas cognitivistas no estudo do ensino. Se bem que, para além das abordagens teóricas e metodológicas oriundas da psicologia cognitivista (36), também outras abordagens, nomeadamente da fenomenologia, trouxeram as suas perspectivas de como explorar este território (13).

A investigação sobre o pensamento do professor foi de início conceptualmente configurada em três domínios distintos, mas coadjuvantes, se entendidos numa relação de interacção cíclica: a) o domínio do planeamento (pensamentos pré e pós interactivos),

b) o domínio dos pensamentos e decisões interactivos e

c) o domínio das teorias e crenças ${ }^{(14)}$.

O planeamento do professor constituiu-se como uma janela estrategicamente privilegiada para contemplar o ensino. Quaisquer intenções de reforma educativa, de inovação curricular ou de ensaio de métodos e materiais didácticos terão que forçosamente passar pelo crivo do planeamento do professor. Compreender o planeamento do professor é, do ponto de vista psicológico, compreender como o professor transforma e interpreta conhecimento, formula intenções e actua em função desse conhecimento e dessas intenções (13). Os teóricos do currículo perceberam a importância desta ideia para o aprofundamento da compreensão da dinâmica da implementação do currículo. De uma fase de estudo do planeamento em separado passou-se a uma nova fase em que ele aparece ligado, entre outros temas, ao estudo da transformação de conhecimento e ao estudo do ensino e aprendizagem das matérias específicas (13). Shavelson (81) considerou a hipótese da tomada de decisão constituir a competência básica do ensino. Adoptar a imagem do ensino como uma cadeia de tomada de decisões pode, no entanto, prestar-se a algumas confusões conceptuais, nomeadamente no que fica entendido por decisão e como se operacionaliza o conceito nos estudos empíricos, muito particularmente no que diz respeito ao processamento da informação na fase interactiva de ensino. Grande parte das acções parecem ser governadas "em piloto automático", na observância de regras e rotinas. Por sua vez, as decisões que pressupõem estudo e ponderação parecem ocupar um lugar modesto no pensamento interactivo do professor. $\mathrm{O}$ conceito de rotina, tradicionalmente associado em Pedagogia à ideia de ausência de vitalidade e inovação no ensino, aparece, com a investigação sobre o pensamento do professor, investido num papel crucial para a explicação do processo de ensino. A rotina corresponde a procedimentos e regras estabelecidos que permitem aos professores e alunos coordenar e controlar sequências de comportamentos. Reduz acentuadamente a carga 
informativa a processar pelo professor e torna previsível ou compreensível o curso da acção para professores e alunos e com isso permite que o professor fique liberto e disponível para outras actividades, nomeadamente a supervisão selectiva do curso da actividade e a implementação de acções não passíveis de se estabelecerem como rotinas. Uma das estratégias para compreender os processos de pensamento do professor foi a de contrastar professores experts ou experientes com professores em início de carreira (em muitos estudos o critério para a classificação de expertise resume-se basicamente ao tempo de serviço).

Constatou-se que os experientes e os novatos apresentam diferenças qualitativas nos processos de pensamento e acção: O processo de pensamento dos professores experientes é mais complexo (45). Estes professores atentam em aspectos diferentes da aula; não se prendem com aspectos superficiais e estímulos irrelevantes (8); requerem mais informação antes de realizar o plano, principalmente sobre as características dos alunos e os materiais e equipamentos disponíveis (44,35); são mais selectivos na utilização da informação durante o planeamento e a interacção; fazem mais uso de rotinas de instrução e gestão (6). Os experts possuem um conhecimento sofisticado da escola, dos alunos, e do ensino que lhes permite tornar mais previsível o decurso da instrução (9). Com a experiência os docentes recorrem mais à memória profissional para a tomada de decisões pré-interactivas (45). Os professores experientes vêm para a situação de instrução não apenas com um plano de actividades e tarefas, mas também com um plano de gestão para implementar essas tarefas e planos para atender a contingências que poderão afectar o progresso da aula (35). A crítica que se faz aos estudos centrados sobre os processos de pensamento e aos estudos comparativos expert-novice (82,11) é a de tenderem a dar pouca atenção ao conhecimento que os professores utilizam para interpretar as situações ou formular os planos e tomar as decisões. A sistematização das diferenças entre experts e novices tende, por sua vez, a realizar-se em torno de generalizações abrangentes sobre a qualidade das diferenças, muitas vezes contribuindo pouco para a compreensão do que os professores sabem. Importa, porém, realçar que foram estes estudos que abriram as portas ao estudo do conhecimento do professor. Os estudos do tipo expert-novice já tinham dado provas noutros domínios, nomeadamente no diagnóstico médico, na cognição política, na resolução de problemas de física, no xadrez e no bridge (11). Três conclusões fundamentais sobre as características do conhecimento do expert ajudam a compreender os seus processos cognitivos:

a) o conhecimento dos experts é especializado, é específico do domínio de expertise considerado;

b) o conhecimento dos experts é organizado;

c) muito do conhecimento dos experts é tácito - não é formal ou facilmente traduzível para instrução directa.

Estas características do conhecimento do expert apontam para a necessidade de estudar melhor a organização e substância do domínio específico do conhecimento e os processos pelos quais esse conhecimento é apreendido. E de uma forma gradual e natural a investigação sobre o pensamento do professor foi dando cada vez mais atenção e espaço à problematização do conhecimento do professor (11).

\section{O CONHECIMENTO DO PROFESSOR}

A questão do conhecimento do professor constitui-se como um ponto de confluência e de debate quer de perspectivas conceptuais e metodológicas (empíricoanalíticas, interpretativistas e social críticas), quer de diferentes parentescos disciplinares (psicologia behaviorista ou cognitivista, sociologia, antropologia), quer ainda das diferentes áreas da investigação pedagógica (currículo, ensino, formação de professores, socialização profissional). A investigação relativa ao conhecimento do professor de uma forma geral não está tanto interessada em identificar o conhecimento formal ou disciplinar e avaliar em que medida o professor domina esse conhecimento, mas tende a preferir considerar o conhecimento a partir da perspectiva pessoal do professor $(24,76)$, do cruzamento do conhecimento com as suas crenças e valores $(16,25,28,60)$, das características distintivas das suas estruturas cognitivas $(27,43,52)$, dos problemas práticos (79) ou dos dilemas (48) que tem de resolver, da orientação e organização que dá ao trabalho e às 
relações na aula (21) e das transformações que tem de realizar sobre o conteúdo para atender às particularidades dos seus alunos (83).

Numa perspectiva constructivista, as novas aprendizagens constroem-se com base no conhecimento anterior que o sujeito possui. Esse conhecimento (onde se inclui também as concepções ingénuas e erros conceptuais) serve de filtro à nova informação. De acordo com o postulado proposto por Chinn e Brewer (12), quando alguém é confrontado com dados que contradizem uma teoria pessoalmente sustentada pode responder basicamente de 7 diferentes maneiras:

a) ignorar os dados anómalos;

b) rejeitar os dados;

c) excluir os dados do domínio da teoria;

d) manter os dados em "suspenso";

e) reinterpretar os dados, conservando a teoria;

f) reinterpretar os dados e introduzir mudanças periféricas na teoria; ou

g) aceitar os dados e mudar a teoria.

A aprendizagem envolve uma interacção entre uma nova concepção e outra já existente, cujo resultado depende da natureza desta interacção. Se houver possibilidade de conciliação entre as concepções, a aprendizagem processa-se sem dificuldade. Se tal não for o caso, a aprendizagem exigirá a restruturação da concepção existente ou mesmo a mudança para uma nova concepção (41). O tema do conhecimento pedagógico do conteúdo mereceu uma atenção especial na Educação Física $(1,32,37,56)$ e tem enquadrado a investigação de diversos autores (29, 31, 78, 94). Inez Rovegno tem desenvolvido um programa de investigação orientado por uma concepção constructivista da aquisição e transformação do conhecimento pedagógico do conteúdo e do conhecimento curricular. De acordo com a autora (68, $69,70,71,72,73,74,75)$, a adesão a uma abordagem constructivista do ensino reclama mudanças em larga escala no conhecimento do professor sobre o conteúdo, a aprendizagem, o ensino e a gestão e requer um apoio prolongado no tempo e uma redução dos obstáculos no interior do contexto da escola e implica concomitantemente um envolvimento afectivo forte com a abordagem e um conjunto de disposições que assegurem e reforcem essa adesão. Sebren (80) pôs em evidência o problema do conhecimento curricular da matéria, quando verificou que alguns candidatos a professor de Educação Física, durante as experiências de prática pedagógica, revelavam não possuir uma visão da matéria como um todo - cada aula era uma unidade discreta desconectada das aulas anteriores e das aulas seguintes. Verificou também a ausência de uma ideia concreta dos níveis de habilidade e de experiência dos alunos na matéria. Todos os candidatos estabeleceram expectativas mais elevadas sobre a capacidade dos alunos.

As crenças acerca da matéria desempenham um papel decisivo na hierarquização dos graus de importância das diferentes componentes da matéria, na especificação do que é importante conhecer. O grande problema das crenças é a sua inércia. É extremamente difícil demover as crenças instaladas, principalmente quando elas resultam de uma longa experiência. Ennis (25) refere que os professores podem despender um grande esforço para encontrar formas de ensinar conteúdos que acreditam ser importantes para os alunos mas, inversamente, investem pouco quando avaliam o conteúdo como pouco importante. Curtner-Smith (16) pôs em relevo os factores que condicionam a resposta dos professores de educação física às propostas de reforma curricular e conclui da ineficácia do movimento da reforma na alteração dos valores e crenças dos professores.

As concepções que os professores possuem acerca dos conteúdos de ensino e acerca dos alunos com quem trabalham reflectem-se no modo como pensam e desenvolvem as suas práticas de ensino. $\mathrm{O}$ conhecimento que o professor tem da disciplina que lecciona interage com conhecimentos, convicções e crenças acerca da educação, do ensino e aprendizagem, acerca dos alunos e acerca dos contextos educativos.

\section{CONCLUSÃO}

Como o título pretende ilustrar, este trabalho não pretende mais do que abrir as portadas de uma das janelas da casa da pedagogia do desporto - a janela da investigação empírica sobre o ensino da educação da educação física. Apesar da sua juventude como 
área de investigação a Pedagogia do Desporto é hoje um edifício muito complexo. Não se esgota na Educação Física, tem como campo de estudos todas as práticas desportivo-corporais e interessa-se pelo praticante dessas actividades de todas as idades e condições. Ainda assim vários temas e perspectivas da investigação pedagógica da educação física são focados muito superficialmente ou não são pura e simplesmente tratados. Estão nestes casos, por exemplo, a investigação sobre a socialização do professor, a investigação sobre as atitudes, as crenças e os processos cognitivos dos alunos, os estudos de orientação social crítica. as metodologias e os métodos de investigação . A literatura recenseada não pretendeu ser exaustiva, antes procurou ser ilustrativa e principalmente compreensiva, incidindo particularmente sobre artigos de revisão, com a intenção de encaminhar os leitores para fontes de recolha de informação mais ricas em extensão e enfoque temático.

\section{REFERÊNCIAS BIBLIOGRÁFICAS}

Bain L (1990). Physical education teacher education. In: Houston R, Haberman M, Sikula J (eds) Handbook of research on teacher education. New York: MacMillan, 758-781 Bain L (1996). History of sport pedagogy in north america. In: Schempp P (ed) Scientific development of sport pedagogy. Munster/New York: Waxmann, 15-40

Ball D (1991). Research on teaching mathematics: making subject-matter part of the equation. In: Brophy J (ed) Advances in research on teaching, vol 2. Greenwich, Connecticut: JAI Press Inc, $1-48$

Bento JO (1995).O outro lado do desporto. Porto: Campo das Letras

Berliner D (1979). Tempus educare. In: Peterson P, Walberg H (eds) Research in teaching: concepts, findings and implication. Burkeley, Calif: McCutchan, 120-135

Borko H, Livingston C (1989). Cognition and improvisation: differences in mathematics instruction by expert and novice teachers. American Educational Research Journal 26:473-498 Brophy J, Good T (1986). Teacher behavior and student achievement. In: Wittrok M (ed) Handbook of research on teaching. New York: MacMillan Publishing Company, 328-375 Byra M, Sherman M (1993). Preactive and interactive decisionmaking tendencies of less and more experienced preservice teachers. Research Quarterly for Exercise and Sport 64:46-55 Calderhead J (1984). Teachers' classroom decision-making.
London: Holt, Rinehart and Winston

Carreiro da Costa F (1995). Condições e factores de ensinoaprendizagem e condutas motoras significativas: uma análise a partir da investigação realizada em portugal VI Congresso galego de educación física.Corunha

Carter K (1990). Teachers knowledge and learning to teach. In: Houston R, Haberman M, Sikula J (eds) Handbook of research on teacher education: MacMillan

Chinn C, Brewer W (1993). The role of anomalous data in knowledge acquisition: a theoretical framework and implications for science instruction. Review of Educational Research 63:1-49

Clark C, Dunn S (1991). Second-generation research on teachers' planning, intentions and routines. In: Waxman $\mathrm{H}$ Walberg $\mathrm{H}$ (eds) Effective teaching: current research. Berkeley, CA: Mccutchan Publishing Corporation, 183-201

Clark C, Peterson P (1986). Teachers' thought processes. In: Wittrok M (ed) Handbook of research on teaching. New York: MacMillan Publishing Company, 255-294

Clark C, Yinger R (1979). Teachers' thinking. In: Peterson P, Walberg H (eds) Research on teaching: concepts, findings and implications. Berkeley, CA: Mccutchan

Curtner-Smith M (1999). The more things change the more they stay the same: factors influencing teachers' interpretations and delivery of national curriculum physical 
education. Sport, Education and Society 4:75-97

Denham C, Lieberman A (1980).Time to learn .Washington

DC: National Institute of Education

Doyle W (1983). Academic work. Review of Educational

Research 53:159-199

Doyle W (1986). Classroom organization and management. In Wittrok M (ed) Handbook of research on teaching. New York: MacMillan Publishing Company, 392-431

Doyle W (1990). Themes in teacher education research. In: Houston R, Haberman M, Sikula J (eds) Handbook of research on teacher education. New York: MacMillan

Doyle W (1992). Curriculum and pedagogy. In: Jackson P (ed) Handbook of research on curriculum: A project of the American Educational Research Association. New York: Macmillan Publishing Company

Dunkin M, Biddle B (1974). The study of teaching. New York: Holt: Rinehart \& Winston

Dussault G (1973). La recherche sur l'enseignement: perspectives historiques et epistémologiques. In: Dussault G Leclerc M, Brunelle J, Turcotte C (eds) L'analyse de l'enseignement. Montréal: Les Presses de l'Université du Québec, 13-35

Elbaz F (1991). Research on teacher's knowledge: the evolution of a discourse. Journal of Curriculum Studies 23:1-19 Ennis C (1994). Knowledge and beliefs underlying curricular expertise. Quest 46:164-175

Ennis C (1995). Teachers' responses to noncompliant students: the realities and consequences of a negotiated curriculum. Teaching \& Teacher Education 11:445-460 Ennis C, Mueller L, Zhu W (1991). Description of knowledge structures within a concept based curriculum framework. Research Quarterly for Exercise and Sport 62:309-318 Fernandez-Balboa J (1991). Beliefs, interactive thoughts, and actions of physical education student teachers regarding pupil misbehaviors. Journal of Teaching in Physical Education 11:59-78 Graber K (1995). The influence of teacher education programs on the beliefs of student teachers: general pedagogical knowledge, pedagogical content knowledge, and teacher education course work. Journal of Teaching in Physical Education 14:157-178

Graça A (1991). O tempo e a oportunidade para aprender o basquetebol na escola: análise de uma unidade de ensino com alunos do $5^{\circ}$ ano de escolaridade. Dissertação apresentada às Provas de Aptidão Pedagógica e de Capacidade Científica..Universidade do Porto: Faculdade de Ciências do desporto e de Educação Física

Graça A (1997). O conhecimento pedagógico do conteúdo no ensino do basquetebol. Dissertação de

Doutoramento.Universidade do Porto: Faculdade de Ciências do desporto e de Educação Física

Graça A (1999). O conhecimento do professor de educação física. In: Bento JO, Garcia R, Graça A (eds) Contextos da pedagogia do desporto. Lisboa: Livros Horizonte, 167-263 Graham G, Heimerer E (1981). Research on teacher effectiveness: a summary with implications for teaching. Quest 33:14-25

Graham K (1987). A description of academic work and student performance during a middle school volleyball unit. Journal of Teaching in Physical Education 7:22-37

Griffey D, Housner L (1991). Differences between experienced and inexperienced teacher's planning decisions, interactions, student engagement, and instructional climate. Research
Quarterly for Exercise and Sport 62:196-204

Griffey D, Housner L (1996). The study of teacher cognition in sport pedagogy. In: Schempp P (ed) Scientific development of sport pedagogy. Munster/New York: Waxmann, 103-122 Griffin L, Dodds P, Rovegno I (1996). Pedagogical content knowledge for teachers: integrate everything you know to help students learn. JOPERD Journal of Physical Educadion Recreation and Dance 67:58-61

Hastie P, Pickwell A (1996). Take your partners: a description of a student social system in a secondary school dance class. Journal of Teaching in Physical Education 15:171-187 Hastie P, Saunders S (1992). A study of task systems and accountability in an elite junior sports setting. Journal of Teaching in Physical Education 11:376-388

Hastie, P, Siedentop, D (1999). An ecological perspective on physical education. European Physical Education Review 5:9-29 Hewson P, Hewson M (1989). Analysis and use of task for identifying conceptions of teaching science. Journal of Education for Teaching 15:191-209

Housner L (1990). Selecting master teachers: evidence from process-product research. Journal of Teaching in Physical Education 9:201-226

Housner L, Gomez R, Griffey D (1993). Pedagogical knowledge structures in prospective teachers - relationships to performance in a teaching methodology course. Research Quarterly for Exercise and Sport 64:167-177

Housner L, Griffey D (1985). Teacher cognition: differences in planning and decision making between experienced and inexperienced teachers. Research Quarterly for Exercise and Sport 56:45-53

Januário C (1994). A influência da experiência profissional nas decisões pré-interactivas e no comportamento interactivo de ensino. Revista Portuguesa de Pedagogia 28:61-78

Januário C (1996). Do pensamento do professor à sala de aula. Coimbra: Livraria Almedina

Jones D (1992). Analysis of task systems in elementary physical education classes. Journal of Teaching in Physica Education 11:411-425

Lampert M (1989). Choosing and using mathematical tools in classroom discourse. In: Brophy J (ed) Advances in research on teaching, vol 1.Greenwich, Co: Jai Press, 223-264

Lawson H (1990). Sport pedagogy research: from informationgathering to useful knowledge. Journal of Teaching in Physical Education 10:1-20

Lee A (1996). How the field evolved. In: Silverman S, Ennis C (eds) Student learning in physical education: applying research to enhance instruction. Champaign, Illinois: Human Kinetics, 9-33 Locke L (1981). Teaching and learning processes in physical activity: the central problem of sport pedagogy. In: Haag $\mathrm{H}$ (ed) XXII ICHPER world congress.Kiel: Schorndorf, Verlag Karl Hoffmann, 140-154

Lynn S (1994). A comparison of pedagogical knowledge structures of preservice students and teacher educators in two institutions. Journal of Teaching in Physical Education 13:140-162

Medley D (1979). The effectiveness of teachers. In: Peterson P, Walberg H (eds) Research on teaching: concepts, findings and implications. Berkeley, CA: Mccutchan, 11-27

Needels M, Gage N (1991). Essence and accident in processproduct research on teaching. In: Peterson P, Walberg $\mathrm{H}$ (eds) Effective teaching: current research. Berkeley, CA: Mccutchan Publishing Corporation, 3-31

Nixon J, Locke L (1973). Research on teaching in physical 
education. In: Travers M (ed) Handbook of research on teaching. Chicago: Rand McNally, 1210-1242

O'Sullivan M, Doutis P (1994). Research on expertise: guideposts for expertise and teacher education in physical education. Quest 46:176-185

Piéron M (1994). Sport pedagogy: highlights on research on teaching research on teacher preparation. Liège: Université de Liège \& AIESEP

Piéron M, Haan J (1981). Interactions between teachers and students in physical education setting: observations of student behaviors. In: Haag H (ed) XXII ICHPER world congress.Kiel: Schorndorf, Verlag Karl Hoffmann, 364-368

Placek J (1983). Conceptions of success in teaching: Busy, happy and good? In: Templin TJ, Olson JK (eds) Teaching in Physical Education. Champaign, Ill: Human Kinetics, 46-56 Placek J, Dodds P (1988). A critical incident study of preservice teachers' beliefs about teaching success and nonsuccess. Research Quarterly for Exercise \& Sport 59:351-358 Postic M (1979). Observação e formação de professores. Coimbra: Livraria Almedina

Rink J (1985). Teaching physical education for learning. St Louis: Times Mirror / Mosby College Publishing Rink J (1999).What do students learn in physical education and how do they learn?. AIESEP Newsletter 63:2-10 Rink J, French K, Lee A, Solmon M, Lynn S (1994). A comparison of pedagogical knowledge structures of preservice students and teacher educators in two institutions. Journal of Teaching in Physical Education 13:140-162

Rosenshine B (1979). Content, time and direct instruction. In: Peterson P, Walberg H (eds) Research on teaching: concepts, findings and implications. Berkeley, CA: Mccutchan, 28-56 Rosenshine B, Furst N (1973). The use of direct instruction to study teaching. In: Travers R (ed) Handbook of research on teaching. Chicago: Rand-McNally, 122-183

Rosenshine B, Stevens R (1986). Teaching functions. In:

Wittrock M (ed) Handbook of research on teaching. New York: Macmillan Publishing Company, 376-391

Rovegno I (1991). A participant-observation study of knowledge restructuring in a field-based physical education methods course. Research Quarterly for Exercise and Sport 62:205-212

Rovegno I (1992). Learning a new curricular approach: mechanisms of knowledge acquisition in preservice teachers. Teaching and Teacher Education 8:253-264 Rovegno I (1992). Learning to teach in a field-based methods course; the development of pedagogical content knowledge. Teacher and Teacher Education 8:69-82

Rovegno I (1993). Content knowledge acquisition during undergraduate teacher education: overcoming cultural templates and learning through practice. American Educational Research Journal 30:611-642

Rovegno I (1993). The development of curricular knowledge: a case of problematic pedagogical content knowledge during advanced knowledge acquisition. Research Quarterly for Exercise and Sport 64:56-68

Rovegno I (1995). Theoretical perspectives on knowledge and learning and a student teacher's pedagogical knowledge of dividing and sequencing subject matter. Journal of Teaching in Physical Education 14:284-304

Rovegno I (1998). The development of in-service teachers' knowledge of a constructivist approach to physical education: teaching beyond activities. Research Quarterly for Exercise and
Sport 69:147-162

Rovegno I, Bandhauer D (1997). Psychological dispositions that facilitated and sustained the development of knowledge of a constructivist approach to physical education. Journal of Teaching in Physical Education 16:136-154

Schempp P (1993). Constructing professional knowledge: a case study of an experienced high school teacher. Journal of Teaching in Physical Education 13:2-23

Schempp P, De Marco G (1996). Instructional theory in sport pedagogy (1994-1995). International Journal of Physical Education 33:5-8

Schempp P, Manross D, Tan S, Fincher M (1998). Subject expertise and teachers' knowledge. Journal of Teaching in Phisical Education 17:342-356

Schon D (1983). The reflective practitioner: how professionals think in action. New York: Basic Books

Sebren A (1995). Preservice teachers' reflections and knowledge development in a field-based elementary physical education method course. Journal of Teaching in Physical Education 14:262-283

Shavelson R (1973). The basic teaching skill: decision making .Stanford CA: Stanford University, School of Education, Center for R \& D in Teaching

Shulman L (1986). Paradigms and research programs in the study of teaching: a contemporary perspective. In: Wittrok M (ed) Handbook of research on teaching. New York: MacMillan Publishing Company, 3- 36

Shulman L (1986). Those who understand: knowledge growth in teaching. Educational Researcher 15:4-14

Siedentop D (1983). Developing teaching skills in physical education, 2nd edn. Palo Alto: Mayfield Publishing Company Siedentop D (1990). Commentary: the world according to Newell. Quest 42:315-322

Siedentop D (1991). Teacher education: types of questions and some answers. In: Park R, Eckert H (eds) New possibilities, new paradigms?. Champaign, Il: Human Kinetics Publishers, 93-99 Siedentop D, Birdwell D, Metzler M (1979). A Process Approach to Measuring Teaching Effectiveness in Physical Education. New Orleans: AAPHERD Annual Conference. Siedentop D, Tousignant M, Parker M (1982). Academic Learning Time-Physical Education Coding Manual. Columbus. Ohio: The Ohio State University

Silverman S (1991). Research on teaching in physical education. Research Quarterly for Exercise and Sport 62:352-364 Silverman S, Ennis C (1996). Student learning in physical education: applying research to enhance instruction .Champaign, Illinois: Human Kinetics

Tinning R, Siedentop D (1985). The characteristics of tasks and accountability in student teaching. Journal of Teaching in Physical Education 4:286-299

Tousignant M, Brunelle J (1982). What we have learned from students and how we can use it to improve curriculum and teaching, learning from students. In: Pieron M, Cheffers J (eds) Studying the teaching in physical education. Liege, Belgium: International Association for Physical Education in Higher Education

Tousignant M, Siedentop D (1983). A qualitative analysis of task structure in required secondary physical education classes. Journal of Teaching in Physical Education 3 Walkwitz E, Lee A (1992). The role of teacher knowledge in elementary physical education instruction: an exploratory study. Research Quarterly for Exercise and Sport 63:179-185 